\title{
Standardization of thromboelastography: a report from the TEG-ROTEM working group
}

\author{
M. CHITLUR, * B. SORENSEN, $\dagger$ G. E. RIVARD, + G. YOUNG, $\mathbb{S}$ J. INGERSLEV, $\uparrow$ M. OTHMAN, ** \\ D. NUGENT, $\dagger+$ G. KENET, + M. ESCOBAR $\mathbb{S}$ and J. LUSHER $\uparrow$ \\ *Division of Hematology/Oncology, Carman and Ann Adams Department of Pediatrics, Wayne State University/Children's \\ Hospital of Michigan, Detroit, MI, USA; †Haemostasis Research Unit, Centre for Haemostasis \& Thrombosis, Guy's \& St \\ Thomas' NHS Foundation Trust, St Thomas' Hospital, London, UK; †Division of Hematology, Hospital Ste Justine, Montreal, \\ QC, Canada; \$Department of Hematology/Oncology, Childrens Hospital Los Angeles, Los Angeles, CA, USA; $\uparrow$ Center for \\ Hemophilia and Thrombosis, Department of Biochemistry, University Hospital Skejby, Aarhus, Denmark; *Department of \\ Pathology and Molecular Medicine, Queens University, Kingston, ON, Canada; ††Division of Hematology, Children's Hospital \\ of Orange County, Orange, CA, USA; +\$Thrombosis Unit, The National Hemophilia Center, Sheba Medical Center, Tel \\ Hashomer, Israel; $\mathbb{S} \mathbb{S}$ Gulf States Hemophilia and Thrombophilia Center, University of Texas Health Science Center at Houston, \\ Houston, TX, USA; and Hemostasis-Thrombosis Program and Coagulation Labs, Division of Hematology/Oncology, \\ Department of Pediatrics, Marion I. Barnhart Chair in Hemostasis Research, Wayne State University, Children's Hospital of \\ Michigan, Detroit, MI, USA
}

Summary. Laboratory evaluation of bleeding disorders has been performed with the standard clotting assays such as the PT and PTT for several decades. Our improved understanding of the process of blood coagulation has now revealed the important role played by the cellular elements such as platelets, monocytes and red blood cells. The need for a test that can assess clotting in a more 'global' manner, beyond the initiation of clot formation, has led to greater interest in assays such as thrombin generation and thromboelastography. Even though there are several publications using thromboelastography it remains a research tool as the methodology is not standardized. In an attempt to show reproducibility and consistency using thromboelastography, a group of investigators from different countries joined hands to form the TEG-ROTEM Working Group. Two studies were performed using PRP and FVIII deficient plasma and an intrinsic pathway activator. This article summarizes the results of the first international effort at standardization of thromboelastography. Both of the instruments using this technology $\left(\right.$ TEG $^{\circledR}$ and ROTEM $\left.^{\circledR}\right)$ were used. Nine laboratories from countries around the globe participated in this effort. The results showed a significant inter-laboratory variance with CV's greater than $10 \%$. Although these results were not satisfactory, this has been the first effort to standardize this methodology and significant work remains to be done to improve reliability and reproducibility. These studies were performed on PRP and the results may be more reliable when preformed on whole blood samples. We believe that it is important to continue this work so that we may investigate the usefulness and potential applications of thromboelastography in the evaluation of bleeding and thrombosis.

Keywords: coagulation, consistency, global assays, reproducibility, standardization, thromboelastography
Correspondence: Meera Chitlur, MD, Carman and Ann Adams Department of Pediatrics, Division of Hematology/Oncology, Wayne State University/Children's Hospital of Michigan, 3901 Beaubien Blvd, Detroit, MI 48201, USA.

Tel.: +313745 5515; fax: +313745 5237;

e-mail:mchitlur@med.wayne.edu

Accepted after revision 28 September 2010

\section{Introduction}

Thromboelastography is a global assay of haemostatic function, from the beginning of clot formation to the end of fibrinolysis. Two systems utilizing this technology include Thrombelastograph/TEG ${ }^{\circledR}$ (Haemonetics, Braintree, MA, USA) and ROTEM (Pentapharm $\mathrm{GmbH}$, Munich, Germany). They are simple to use 
and proven to be effective methods of intraoperative monitoring to optimize blood product selection and utilization and have been shown to improve outcomes during complex surgical procedures such as cardiothoracic surgery and liver transplantation [1,2]. Case reports or small case series have addressed the usefulness of thromboelastography in the management of bleeding or monitoring of treatment with bypassing agents $[3,4]$. In order to establish the usefulness of this test a standardized methodology needs to be developed and reproducibility and consistency have to be demonstrated. In an attempt to initiate this process, the TEGROTEM Working group was established in 2006 and investigators from several countries joined hands to perform these studies. Herein we present the results of the first international study, wherein nine laboratories from six countries (Canada, Denmark, Israel, Norway, UK and USA) participated.

\section{Materials and methods}

The main components of the thromboelastograph consist of a cylindrical cup and a pin. In the TEG ${ }^{\circledR}$ the cup is oscillated through an angle of $4^{\circ} 45^{\prime}$ over a $10 \mathrm{~s}$ interval, while in the ROTEM ${ }^{\circledR}$, the pin oscillates and the cup remains stationary. The torque of the cup is transmitted to the pin through sample viscosity in the cup. Unclotted blood transmits little or no torque from cup to pin. As the blood clots, fibrin fibres form between the cup and pin, transferring a portion of the cup's motion to the pin. With clot lysis the torque decreases. The computerized system automatically records kinetic changes in the whole blood sample. The clots physical properties, such as the rate of clot formation, the strength and stability of the clot are thereby recorded by the system, and are dependent on the interaction of the coagulation factors, platelets and fibrinogen. The tracing produced is called the thromboelastogram as shown in Fig. 1.

The TEG $^{\circledR}$ and ROTEM $^{\circledR}$ although similar have minor differences in the mechanical aspects. This is represented in Table 1.
Even though the parameters are identical there are differences in the diagnostic nomenclatures.

1. Coagulation time: $R$ on $\mathrm{TEG}^{\circledR}$ and $\mathrm{CT}$ on ROTEM $^{\circledR}$.

2. Clot Formation time: $K$ on $\mathrm{TEG}^{\circledR}$ and $\mathrm{CFT}$ on ROTEM $^{\circledR}$.

3. Maximum Clot Firmness: $\mathrm{MA}$ on $\mathrm{TEG}^{\circledR}$ and $\mathrm{MCF}$ on ROTEM ${ }^{\circledR}$

4. Shear Elastic Modulus Strength: $G$ on both instruments.

5. $\alpha /$ Angle-Rate of polymerization of clot: $\alpha$ on both instruments.

While there are several other parameters available on both instruments to measure other parameters of coagulation and clot lysis, the above represent the common parameters used to assess coagulation.

Since the TEG $^{\circledR}$ and ROTEM $^{\circledR}$ are similar yet not identical, the objective of our efforts was to determine if the same instrument could produce similar results when under similar conditions, thereby proving reproducibility and consistency. We did not intend to compare between the two instruments, TEG $^{\circledR}$ and ROTEM $^{\circledR}$.

In order to assess the reproducibility of the thromboelastographic parameters on both the $\mathrm{TEG}^{\circledR}$ and ROTEM $^{\circledR}$ two sets of experiments were conducted using the initiator for the intrinsic pathway provided by the manufacturers of the instruments (Kaolin/Intem) first with platelet rich plasma (PRP) and in the second experiment both normal pooled plasma and factor VIII (FVIII) deficient plasma were evaluated. The object of this study was to establish consistency and reproducibility between laboratories using the same method on the same instrument.

In order to ensure that the instruments were in good working order it was determined that the date of the last maintenance check was within 6 months of running the test.

Since PRP is more stable during storage and transportation, this was determined to be the sample of choice. A pool of normal as well as FVIII deficient plasma was provided by Center for Hemophilia and

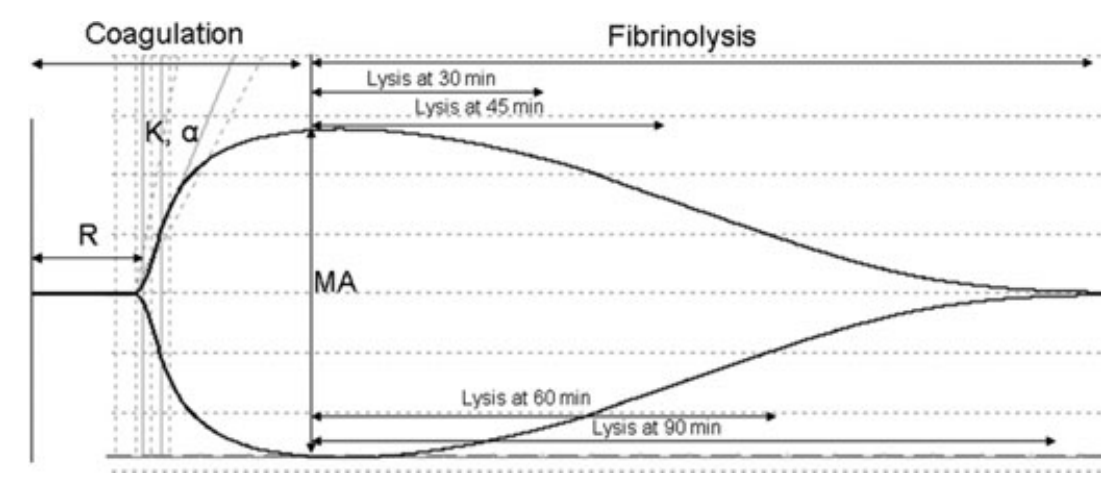

Fig. 1. The thromboelastography trace. 
Table 1. Characteristics of the TEG ${ }^{\circledR}$ and ROTEM ${ }^{\circledR}$.

\begin{tabular}{|c|c|}
\hline TEG & ROTEM \\
\hline Two channels & Four channels \\
\hline $\begin{array}{l}\text { A pin connected with a torsion wire, is inserted into the sample. } \\
\text { Sample cup oscillates }\end{array}$ & $\begin{array}{l}\text { The pin (sensor) is fixed on the tip of a rotating shaft which is guided by a ball } \\
\text { bearing system. The shaft rotates back and forth. Sample cup is stationary }\end{array}$ \\
\hline $\begin{array}{l}\text { Mixing of the contents in the sample cup is done with the oscillation of } \\
\text { the cup over the pin. }\end{array}$ & $\begin{array}{l}\text { Mixing of the contents in the cup is accomplished by re-aspirating into the } \\
\text { automated pipette. }\end{array}$ \\
\hline Activators used: & Activators used: \\
\hline $\begin{array}{l}\text { KAOLIN: Vial coated with kaolin to which } 1 \mathrm{~mL} \\
\text { blood per plasma is added. Volume sufficient to run two channels }\end{array}$ & $\begin{array}{l}\text { INTEM: Partial thromboplastin phospholipids made from rabbit brain. Blood is } \\
\text { not mixed outside the cup therefore only } 400 \mu \mathrm{L} \text { required per test. }\end{array}$ \\
\hline Sample volume: $360 \mu \mathrm{L}$ & Sample volume: $340 \mu \mathrm{L}$ \\
\hline
\end{tabular}
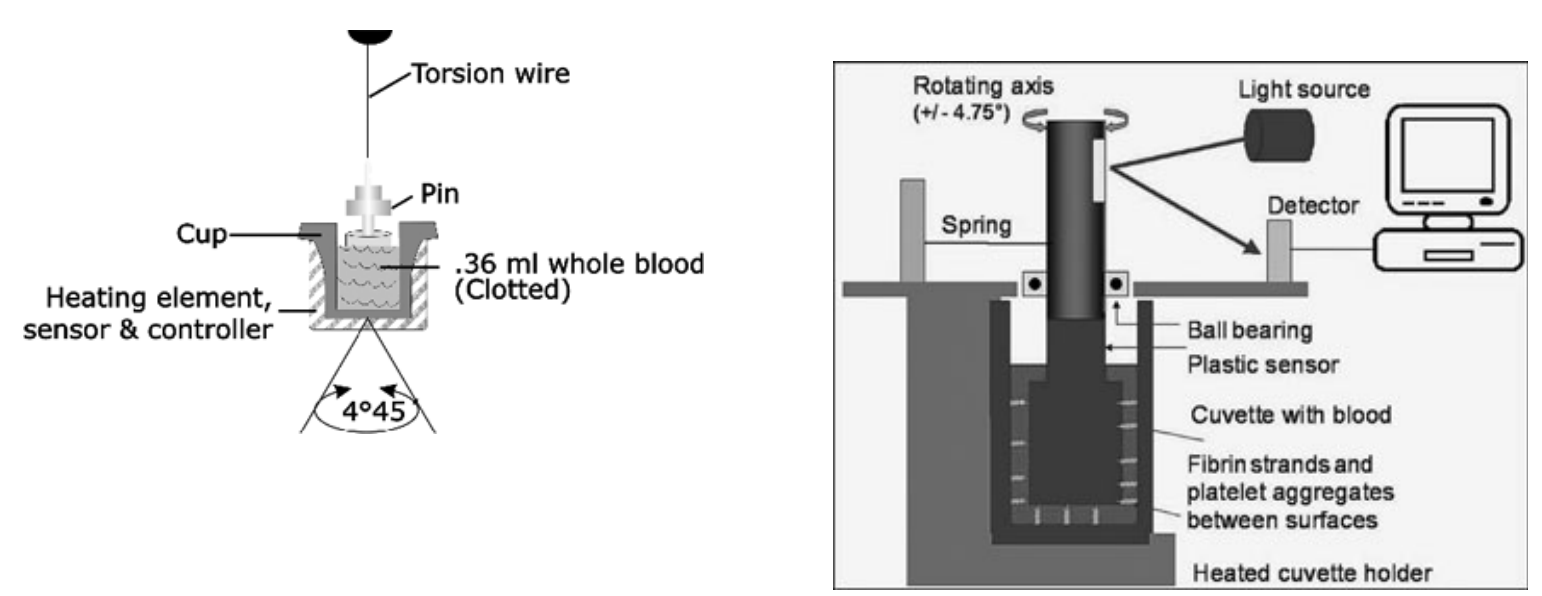

Thrombosis, Aarhus University Hospital, Skejby, Denmark (Ingerslev \& Sorensen). Although whole blood is the sample of choice for these assays, this was a limitation that was foreseen.

Method: Intrinsic activation was evaluated using standard reagents (Same lot number) provided by the manufacturers: Kaolin from Haemoscope and InTEM from Pentapharm. Samples were run using the same protocol as recommended for whole blood by the manufacturer.

For the TEG $^{\circledR}$ : Each aliquot of plasma was thawed for $10 \mathrm{~min}$ in a water bath at $37^{\circ} \mathrm{C}$. One $\mathrm{mL}$ of the plasma was then transferred to the kaolin vial and mixed by inverting it five times. Then $340 \mathrm{~mL}$ of the kaolin/plasma mixture was transferred via reverse pipetting into the TEG cup already containing $20 \mu \mathrm{L}$ of the $\mathrm{CaCl}_{2}$ supplied with kit, and recording was initiated. The temperature was maintained at $37^{\circ} \mathrm{C}$ during the testing. Since this methodology is very sensitive to the technique, reverse pipetting was used for all steps in the testing. All precautions were taken to minimize the appearance of bubbles in the sample cup as this has been noted to activate the sample. All tests were recorded for a minimum of $60 \mathrm{~min}$. The above procedure was repeated twice with the same TEG equipment on three different days for a total of 12 recordings/instrument of $60 \mathrm{~min}$ each.

For the ROTEM $^{\circledR}$ : Each aliquot of plasma was thawed for $10 \mathrm{~min}$ in a water bath at $37^{\circ} \mathrm{C} .300 \mu \mathrm{L}$ of plasma was transferred to ROTEM cup already containing $20 \mu \mathrm{L}$ InTEM and $20 \mu \mathrm{L}$ starTEM (buffered, specifically concentrated calcium chloride solution supplied by manufacturer) and recording was initiated. Temperature was maintained at $37^{\circ} \mathrm{C}$ during the testing. Electronic pipette was preferred to be used for all runs. If manual pipetting was used, reverse pipetting was recommended. All attempts were made to minimize the appearance of bubbles in the sample cup and all tests were run for a minimum of $60 \mathrm{~min}$. The above procedure was repeated twice with the same ROTEM ${ }^{\circledR}$ equipment on three different days for a total of 24 recordings/instrument of $60 \mathrm{~min}$.

Both normal and abnormal plasma were tested in a similar manner.

Parameters normally used to assess the process of coagulation were determined to be: Coagulation time $\left(R\right.$ on $\mathrm{TEG}^{\circledR}$ and CT on $\left.\mathrm{ROTEM}^{\circledR}\right)$, Clot formation time $\left(K\right.$ on TEG $^{\circledR}$ and CFT on ROTEM $\left.^{\circledR}\right)$, Maximum clot firmness (MA on TEG ${ }^{\circledR}$ and MCF on ROTEM $^{\circledR}$ ), Shear Elastic Modulus Strength or clot strength $(G$ on both instruments), and $\alpha /$ Angle-Rate of polymerization of clot: ( $\alpha$ on both instruments) and therefore it would be important to achieve reproducibility in these.

Six assays were conducted for each parameter, for normal as well as abnormal controls. The six assays are split into three sessions of two assays.

Our first task included 5 ROTEM $^{\circledR}$, s and 6 TEG $^{\circledR}$, $s$ from five different laboratories. This study was mainly 
limited by the availability of pooled normal plasma. Our second effort was better organized and included nine laboratories from around the globe and 11 ROTEM $^{(\text {'s }}$ and 6 TEG $^{(\text {) }}$ s.

\section{Statistical Analysis}

Means and SD's for each run and each laboratory were calculated and compared using the Student $t$-test. A co-efficient of variance $(\mathrm{CV})$ was calculated from the means and SD for the entire group. Inter laboratory variance was evaluated using the ANOvA. Each instrument $\left(\right.$ TEG $^{\circledR}$ and ROTEM $^{\circledR}$ ) was formally and individually evaluated in this manner.

\section{Results}

Since the second task included a larger number of laboratories and both PRP and FVIII deficient plasma were used, the results presented below were those obtained in Task 2.

TEG $^{\circledR}$ : The means and SD's for the 28 runs, 14 from each channel are shown in Tables 2 and 3. There were no significant differences between channels.

ROTEM $^{\circledR}$ : The means and SD's from the 120 runs on 5 ROTEM's are shown in the Tables 4 and 5. Thirty

Table 2. TEG $^{\circledR}$ Parameters with PRP.

\begin{tabular}{lccccc}
\hline & $R$ & $K$ & \multicolumn{1}{c}{$A$} & \multicolumn{1}{c}{ MA } & \multicolumn{1}{c}{$G$} \\
\hline Mean & 4.38 & 2.38 & 74.51 & 39.45 & 3.39 \\
SD & 0.74 & 1.43 & 5.26 & 7.74 & 1.1 \\
CV & 17 & 59.98 & 7.06 & 19.62 & 32.55 \\
\hline CV & & & & &
\end{tabular}

Table 3. $\mathrm{TEG}^{\circledR}$ Parameters with factor VIII Deficient Plasma.

\begin{tabular}{lrccrr}
\hline & $R$ & $K$ & $A$ & \multicolumn{1}{c}{ MA } & \multicolumn{1}{c}{$G$} \\
\hline Mean & 28.58 & 16.212 & 24.90 & 32.25 & 2.45 \\
SD & 2.45 & 5.04 & 11.78 & 7.07 & 0.83 \\
CV & 8.58 & 31.14 & 47.29 & 21.91 & 33.88 \\
\hline CV, co-efficient of variance.
\end{tabular}

Table 4. ROTEM $^{\circledR}$ parameters with PRP.

\begin{tabular}{lrrrrr}
\hline & \multicolumn{1}{c}{ CT } & CFT & A & MCF & \multicolumn{1}{c}{ G } \\
\hline Mean & 152.59 & 346.93 & 78.26 & 34.43 & 2690.3 \\
SD & 21.44 & 135.53 & 2.44 & 4.99 & 571.93 \\
CV & 14.05 & 39.07 & 3.12 & 14.49 & 21.26 \\
\hline
\end{tabular}

$\mathrm{SD}$, standard deviation; $\mathrm{CV}$, coefficient of variance.

Table 5. ROTEM $^{\circledR}$ parameters with FVIII deficient plasma.

\begin{tabular}{lrrrrr}
\hline & \multicolumn{1}{c}{ CT } & \multicolumn{1}{c}{ CFT } & \multicolumn{1}{c}{ A } & MCF & \multicolumn{1}{c}{ G } \\
\hline Mean & 487.93 & 373.64 & 45.84 & 37.15 & 3024.7 \\
SD & 64.22 & 191.51 & 7.51 & 5.91 & 752.08 \\
CV & 13.16 & 51.25 & 16.39 & 15.90 & 24.87 \\
\hline
\end{tabular}

$\mathrm{SD}$, standard deviation; $\mathrm{CV}$, coefficient of variance.
Table 6. Reproducibility as demonstrated by the CAP.

\begin{tabular}{lllcc}
\hline & \multicolumn{1}{c}{$\mathrm{R}$} & \multicolumn{1}{c}{$\mathrm{K}$} & \multicolumn{1}{c}{$\mathrm{A}$} & \multicolumn{1}{c}{ MA } \\
\hline Mean & 1.46 & 0.80 & 82.67 & 52.83 \\
SD & 0.26 & 0.03 & 1.47 & 2.95 \\
Range & $0.7-2.3$ & $0.8-1.1$ & $75.3-85.9$ & $42.6-64.4$ \\
\hline
\end{tabular}

$\mathrm{SD}$, standard deviation.

runs each were performed with both PRP and FVIII deficient plasma.

Information on reproducibility as conducted by the American Proficiency Institute and the College of American Pathologists (CAP) is shown in Table 6. These institutions provide proficiency certifications to laboratories to ensure acceptable standards of testing. These are results for the year 2007 and mostly performed by perfusionists, since the TEG is licenced in the US as a point of care instrument to guide transfusion requirements. Table 6 shows excellent reproducibility and the number of laboratories participating in this exercise is significant. However, they are using lyophilized plasma and also there is no initiator and the differences in strength of the initiator do not exist.

An attempt was made to determine if there was a correlation between laboratory variation and the high CV's seen on our results.

As seen in Fig. 2, variables such as CT and MCF which are parameters that are more commonly used have a lower CV, compared to the CFT or $\alpha$ angle.

Similar variations were seen in CV's between laboratories on TEG with FVIII deficient plasma as shown in Fig. 3. The CV's were higher for the $K$ value on the $\mathrm{TEG}^{\circledR}$ which is the same as the CFT on the $\mathrm{ROTEM}^{\circledR}$.

Among the parameters that were measured, it is important to note that the $\mathrm{CV}$ for the R/CT and MA/ MCF were less than $20 \%$. Although these are not acceptable for a routine laboratory assay, these may be related to the differences in the activators or the use of frozen plasma which may be avoided if whole blood is used for the assay. The other parameters such as $K$ and $\alpha$ angle have been known to have increased variability and are also not used often in routine practice for

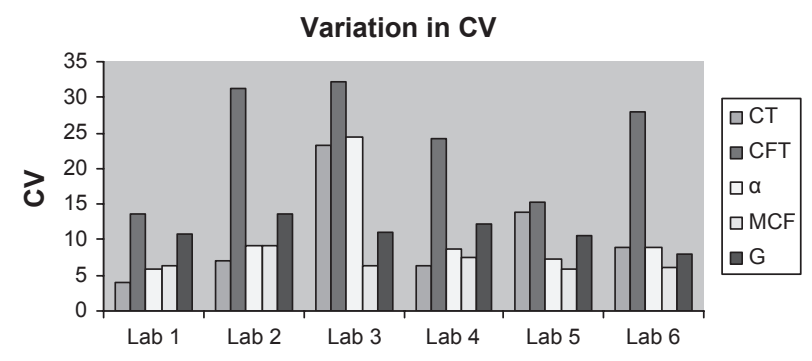

Fig. 2. Variation in CV between laboratories on ROTEM $^{\circledR}$ with FVIII deficient plasma. 


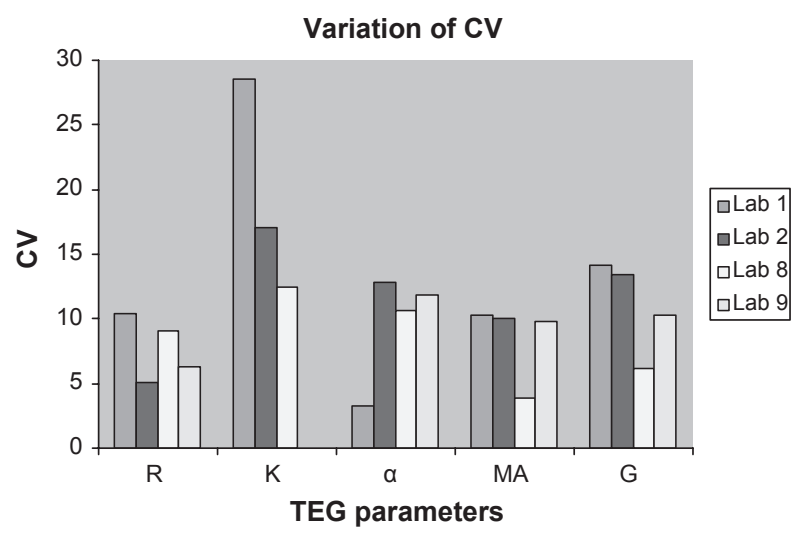

Fig. 3. Variation in $\mathrm{CV}$ between laboratories on $\mathrm{TEG}^{\circledR}$ with FVIII deficient plasma.

clinical judgment. We hope that by using whole blood these CV's may show improvement and probably reach acceptable levels.

\section{Discussion}

Thromboelastography was first developed by Dr. Hellmut Hartert at University of Heidelberg School of Medicine in 1948. It is an elastic modulus based assessment of hemostasis that is gaining popularity in research and clinical settings since the availability of the newly improved analyzers. It is currently used in the point of care management of acute peri-operative bleeding. The two currently available instruments using this principal are the $\mathrm{TEG}^{\circledR}$ and the $\mathrm{ROTEM}^{\circledR}$.

Classical thromboelastography is a global assay of coagulation which allows the rapid assessment of coagulation and fibrinolysis using native whole blood, anticoagulated citrated recalcified whole blood or activated whole blood. Since it does not provide any quantitative analysis of clotting or anticoagulant factors, it remains a research tool in hematology [5]. With the emergence of interest in global assays in coagulation which may provide a more physiological approach to the study of the process of hemostasis, there has been resurgence in interest in tests such as thromboelastography and thrombin generation.

Thromboelastography has now been tested in various clinical scenario such as, perioperatively in cardiac and hepatic surgery and transplantation to decrease the number of transfusions [1,6], as a screening tool for hypercoaguability [7] and recently for the management of bleeding in patients with bleeding disorders $[3,4,8]$. Several recent publications have also attempted to assess hypercoaguability using thromboelastography [7,9]. This method has also been shown to be useful in assessing the bleeding tendency in patients with bleeding disorders $[10,11]$. While it is gaining popularity in the clinical arena, the methodology remains at the discretion of the user/manufacturer. There are a few studies that have attempted to determine the validity and/or reliability of the two instruments, but they have been few and far between $[5,12,13]$.

In an attempt to standardize the methodology and to determine if the tests performed on both instruments are reliable and reproducible when the standard operating procedure as recommended by the manufacturer is used, a TEG-ROTEM Working Group was established in 2006. The results shown in this study were performed by an international group of investigators from several countries, but showed significant variation between laboratories and instruments. Although one study from Germany reports a multicentre (six centres) investigation of results using the ROTEM to be consistent, where the CV's were $\leq 14 \%$ for the CT and CFT [12], we were unable to reproduce this finding. Lang et al. performed the study using whole blood while we used frozen plasma which may have contributed to the variability, as the whole blood assay may be more reproducible compared to the plasma based assay. There was significant variation from laboratory to laboratory in our study indicating that operator variability may play a large role in the results that were obtained.

Thromboelastography is a promising tool for the evaluation of hemostasis but cannot be utilized to its full capacity until studies demonstrate the reliability and reproducibility of the assay. These results show us that there remains a great need for continued efforts to standardize the test, and this has been the first large, collaborative, international effort in this direction. As a group with vested interest in this assay we hope to continue our efforts to standardize the assay and provide useful information to facilitate the use of this test in the assessment of coagulation.

\section{Acknowledgements}

Our sincere thanks to Hemoscope Corp, Niles, IL, USA (Previous manufacturer of the $\mathrm{TEG}^{\circledR}$ ) and Pentapharm, GmbH, Munich, Germany, for providing the disposables and activators for the studies without whose assistance these studies could not have been conducted. We are also extremely grateful to the Center for Hemophilia and Thrombosis, Aarhus University Hospital, Skejby, Denmark (Ingerslev \& Sorensen), for providing the plasma for both studies.

The TEG-ROTEM Working Group includes members other than those in the author list. Our sincere thanks to the other participants in the group without whose efforts these studies would not be possible. They include: David Lillicrap (Canada), Madhvi Rajpurkar (USA), Amit Soni (USA), Edward Tuddenham (UK), Catherine Hayward (Canada), Elizabeth Donnachie (USA) and Ann Ridell (UK).

\section{Disclosures}

The authors stated that they had no interests which might be perceived as posing a conflict or bias. 


\section{References}

1 Shore-Lesserson L, Manspeizer HE, DePerio M, Francis S, Vela-Cantos F, Ergin MA Thromboelastography-guided transfusion algorithm reduces transfusions in complex cardiac surgery. Anesth Analg 1999; 88 312-9.

2 Fuchs RJ, Levin J, Tadel M, Merritt W. Perioperative coagulation management in a patient with afibrinogenemia undergoing liver transplantation. Liver Transpl 2007; 13: 752-6.

3 Yoshioka A, Nishio K, Shima M. Thrombelastgram as a hemostatic monitor during recombinant factor VIIa treatment in hemophilia A patients with inhibitor to factor VIII. Haemostasis 1996; 26(Suppl 1): 139-42.

4 Young G, Blain R, Nakagawa P, Nugent DJ Individualization of bypassing agent treatment for haemophilic patients with inhibi- tors utilizing thromboelastography. Haemophilia 2006; 12: 598-604.

5 Vig S, Chitolie A, Bevan DH, Halliday A, Dormandy J. Thromboelastography: a reliable test? Blood Coagul Fibrinolysis 2001; 12: 555-61.

6 Harding SA, Mallett SV, Peachey TD, Cox DJ. Use of heparinase modified thrombelastography in liver transplantation. Br J Anaesth 1997; 78: 175-9.

7 O'Donnell J, Riddell A, Owens D et al. Role of the Thrombelastograph as an adjunctive test in thrombophilia screening. Blood Coagul Fibrinolysis 2004; 15: 207-11.

8 Hayashi T, Tanaka I, Shima M et al. Unresponsiveness to factor VIII inhibitor bypassing agents during haemostatic treatment for life-threatening massive bleeding in a patient with haemophilia $\mathrm{A}$ and a high responding inhibitor. Haemophilia 2004; 10: 397-400.

9 Hvitfeldt Poulsen L, Christiansen K, Sorensen B, Ingerslev J. Whole blood thrombe- lastographic coagulation profiles using minimal tissue factor activation can display hypercoagulation in thrombosis-prone patients. Scand J Clin Lab Invest 2006; 66: 329-36.

10 Chitlur M, Warrier I, Rajpurkar M et al. Thromboelastography in children with coagulation factor deficiencies. $\mathrm{Br} \mathrm{J} \mathrm{Hae-}$ matol 2008; 142: 250-6.

11 Sorensen B, Ingerslev J. Whole blood clot formation phenotypes in hemophilia A and rare coagulation disorders. Patterns of response to recombinant factor VIIa. $J$ Thromb Haemost 2004; 2: 102-10.

12 Lang T, Bauters A, Braun SL et al. Multicentre investigation on reference ranges for ROTEM thromboelastometry. Blood Coagul Fibrinolysis 2005; 16: 301-10.

13 Nielsen VG. A comparison of the Thrombelastograph and the ROTEM. Blood Coagul Fibrinolysis 2007; 18: 247-52. 